\title{
Data Card
}

National Cancer Institute

\section{Source}

National Cancer Institute. Data Card. NCI Thesaurus. Code C160947.

A card which contains data for automatic processing of an item. 